\title{
COVID-19 disease in children: not as mild as we have been led to believe
}

\author{
Laura Moreno-Galarraga ${ }^{1,2,3}$ [D $\cdot$ Elsie M. Taveras ${ }^{4,5,6}$
}

Received: 26 May 2020 / Accepted: 15 June 2020 / Published online: 23 June 2020

(c) Children's Hospital, Zhejiang University School of Medicine 2020

Since severe acute respiratory syndrome coronavirus-2 (SARS-CoV-2) emerged, we have followed this pandemic with great interest and concern from Europe (NavarraSpain) and the United States (Boston-Massachusetts). We were expecting children to present to emergency departments with the "classical COVID-19 symptoms-triad" such as fever, cough, and a range of respiratory distress [1] and we had learned from countries affected earlier by the virus, that it appeared to be less common and relatively mild in children [2,3].

However, in April 2020, pediatricians from different Europeans countries and the United States began reporting cases of COVID-19 in children, requiring intensive care unit treatment and associating severe cardiac-symptoms, generally presenting 3-5 weeks after the COVID-19 peak. We have learned how children can present with a wide range of clinical symptoms, with respiratory symptoms not always being present and how some previously healthy children can present with skin-lesions, hypotension and tachycardia developing in the next hours an acute cardiac failure (with elevated cardiac-enzymes and imagine-compatible findings with acute-myocarditis).

Laura Moreno-Galarraga

laura.moreno.galarraga@cfnavarra.es

1 Department of Pediatrics, CHN, Complejo Hospitalario de Navarra, Servicio Navarro de Salud, C/Irunlarrea 3, 31080 Pamplona, Spain

2 IdiSNA, Navarra Institute for Health Research, Pamplona, Spain

3 Department of Preventive Medicine and Public Health, School of Medicine, University of Navarra, Pamplona, Spain

4 Division of General Academic Pediatrics, Massachusetts General Hospital for Children, Boston, MA, USA

5 Department of Pediatrics, Harvard Medical School, Boston, MA, USA

6 Department of Nutrition, Harvard T.H.Chan School of Public Health, Boston, MA, USA
As more case-reports and publications emerge in the next months, with different countries reporting their cases, we expect to learn more about this severe pediatric presentation, also referred as "Kawasaki-like" or "pediatric inflammatory multisystem syndrome". We believe it is related to coronavirus but it seems to affect in a late phase of the disease [many cases report negative polymerase chain reaction (PCR) but positive immunoglobulin G], therefore, it might be related to a systemic inflammatory response or a delayed immune response. Pediatricians should be aware of this uncommon but possible complication, tachycardia and hypotension should be used as early red-flags, and SARS-CoV-2 should be suspected even if PCR is negative.

Many questions still need answers, but if this new pediatric presentation is related to COVID-19, it deserves fast and deeper investigation. The association between pediatricsevere-cardiac-cases and SARS-CoV-2 needs to be demonstrated and best prevention strategies, red flags, diagnosis tools and treatments need to be established. A global response should be taken to address a global problem. A global health institution, as the World Health Organization, might be a wise option to quickly recollect and analyze all reported cases, to better understand this new COVID-19 presentation in children. Global data recollection might help establish the correct guidelines to prevent, early detect and effectively treat pediatric-severe-cardiac-cases worldwide.

Author contributions MGL conceptualized the idea, drafted the initial manuscript, and revised the manuscript. TEM reviewed and revised the manuscript. All authors have agreed to submit this article in the present form. Each has participated sufficiently in the conception and design of the work. All authors approved the final manuscript as submitted and agree to be accountable for all aspects of the work.

Funding The Spanish Association of Pediatrics Foundation supported the study (INVEST-AEP-2018 09-18 02-19).

\section{Compliance with ethical standards}

Ethical approval Not needed. 
Conflict of interest MGL received a grant, from the Spanish Association of Pediatrics to collaborate with TEM on pediatric research at the Division of General Academic Pediatrics, Massachusetts General Hospital for Children, Boston, MA during 2018-19.

\section{References}

1. Guan WJ, Ni ZY, Hu Y, Liang WH, Ou CQ, He JX, et al. Clinical characteristics of coronavirus disease 2019 in China. N Engl J Med. 2020;382:1708-20.
2. Li Q, Guan XH, Wu P, Wang XY, Zhou L, Tong YQ, et al. Early transmission dynamics in Wuhan, China, of novel coronavirusinfected pneumonia. N Engl J Med. 2020;382:1199-207.

3. Wu ZY, McGoogan JM. Characteristics of and important lessons from the coronavirus disease 2019 (COVID-19) outbreak in China: summary of a report of 72314 cases from the Chinese Center for Disease Control and Prevention. JAMA. 2020;323:1239-42.

Publisher's Note Springer Nature remains neutral with regard to jurisdictional claims in published maps and institutional affiliations. 